Editorial

Forum Kind Jugend Sport 2021 2 2:91-92

https://doi.org/10.1007/s43594-021-00041-w

Angenommen: 6. Oktober 2021

๑c Der/die Autor(en) 2021

\author{
Stefan Raid' $\cdot$ Nils Neuber ${ }^{2} \cdot$ Peter Lautenbach ${ }^{3}$ \\ 'Deutsche Sportjugend, Hamburg, Deutschland \\ ${ }^{2}$ Westfälische Wilhelms-Universität Münster, Münster, Deutschland \\ ${ }^{3}$ Deutsche Sportjugend, Frankfurt am Main, Deutschland
}

\title{
„Die Medaille hat zwei Seiten“
}

früherer Geschäftsführer der Deutschen Sportjugend (dsj). Er setzt weit vor der Pandemie an und findet, dass die schon länger bestehenden WHO-Empfehlungen zur Bewegungszeit junger Menschen zu einfach gedacht sind. Sein Kommentar könnte der Anstoß zu einer Debatte sein. Die Botschaft: Sechzig Minuten Bewegung am Tag sind gut - aber diese rein quantitative Forderung reduziert die Diskussion über das Wohlbefinden der Jugend auf die motorischen Defizite. All das, was Sport für Kinder und Jugendliche darüber hinaus bedeutet - soziale Interaktion, Freundschaften, Streit, eigenes Engagement und vieles andere mehr -, fällt unter den Tisch.

Womit wir bei der Rolle der Sportvereine sind und einem Tipp für sie: Setzt auch auf die soziale Karte! Neben motorischen Inhalten von Training und Wettkampf trifft vor allem die soziale Ebene im Verein die Bedürfnisse der jungen Generation. Das ist eine Erkenntnis des Forschungsbeitrags von Nils Neuber, Stefanie Dahl und Sebastian Salomon in dieser Ausgabe. Die Wissenschaftler*innen der Westfälischen Wilhelms-Universität Münster haben Entfaltungs- und Entwicklungspotenziale von Jugendlichen verglichen, dabei auf Stichproben aus den Jahren 2002 und 2019 zurückgegriffen und erkennen eine Tendenz vom spielerischen Sporterleben in der Freizeit zur körper- und fitnessbezogenen Selbstoptimierung. Eines ihrer Fazits: Schul- und Vereinssport könnten bewusster das soziale Potenzial nutzen, das der Sport in der Gruppe bietet.

Im Sinne des Wissenschaft-PraxisDialogansatzes von Forum Kinder- und Jugendsport haben wir dazu zum einen den Würzburger Bildungsforscher Heinz Reinders befragt, zum anderen mit Leo- nard Döring, Karolin Huse, Max Sormani und Paul Werner vier junge Engagierte aus den Strukturen des gemeinnützigen, organisierten Sports - eine lebendige Diskussionsrunde mit interessanten Einblicken von Mittzwanzigern, die gleichermaßen für Jugendlichkeit wie den organisierten Sport stehen.

Nochmal ein Schritt zurück, um nicht missverstanden zu werden: Daten zur motorischen Entwicklung der Jugend im Längsschnitt sind wichtig. Ohne eine differenzierte Analyse geht es nicht. Das zeigt ein weiterer Forschungsbeitrag, in dem Anke HanssenDoose und Kolleg*innen von der Pädagogischen Hochschule Karlsruhe und dem Karlsruher Institut für Technologie anschaulich darstellen, wie der Sportverein die motorische Leistungsfähigkeit der Kinder und Jugendlichen beeinflusst. Den Dialogbeitrag dazu bildet ein Essay der Karlsruher Sportwissenschaftlerin Katja Klemm. Sie schildert, wie die Corona-Zeit und die daraus getroffenen Entscheidungen für den Sport die Gesundheit der Kinder und Jugendlichen in Deutschland aufs Spiel setzt und wie Sport, Spiel und Bewegung helfen können. Passend zur aktuellen Situation der Kinder und Jugendlichen ist auch die Arbeit von Roland Naul vom Willibald Gebhardt-Institut, der in einem Beitrag zur Forschung deutsche und europäische Studien $\mathrm{zu}$ aktivem Sporttreiben und passiven Bildschirmzeiten in der Pandemie miteinander vergleicht.

Abgerundet wird diese vierte Ausgabe von Forum Kinder- und Jugendsport durch Fachbeiträge zu verschiedenen Themen: In der Rubrik ,aktuelles Stichwort" skizziert Ralf Lippmann, Lehr- und Prüfungsreferent des Deutschen JudoBundes, was Fairness in seiner Sportart 
bedeutet und dass erst der passende Umgang mit der eigenen Niederlage den persönlichen Sieg möglich macht. Dominik Edelhoff (Universität DuisburgEssen) schildert die Idee einer sportpädagogischen Entwicklungsforschung am Beispiel des Projekts „Open Sunday“, das mit offenen Hallen Kinder des unmittelbaren Sozialraums zu Bewegung, Spiel und Sport einlädt. In der anschließenden Rezension des neu erschienenen OpenSunday-Buchs betrachtet Rolf Schwarz von der Pädagogischen Hochschule Karlsruhe das dahinterliegende Konzept. Axel Dietrich und Dominik Ulrich, Experten aus den Gremien zur Weiterentwicklung der schulsportlichen Wettbewerbe Jugend trainiert für Olympia \& Paralympics und Bundesjugendspiele, erläutern in ihrem Beitrag die Hintergründe der Anpassungen. Juliane Lanz von der Universität Rostock liefert eine Eignungsprüfung des Bouldersports für Kinder und Jugendliche und schließlich erläutert dsj-Referentin Katharina Morlang die Ergebnisse einer Befragung der Mitgliedsorganisationen der Deutschen Sportjugend zur Verankerung von Nachhaltigkeit und einer Bildung für nachhaltige Entwicklung.

Wieder liegt damit ein breites Feld an Themen vor Ihnen, liebe Leserinnen und Leser. Bevor wir den Ring freigeben, möchten wir unser Vorwort nutzen, um ein wichtiges Dankeschön auszusprechen. Denn ohne das wertvolle, freiwillige Engagement unserer Gutachter ${ }^{*}$ innen im Peer-Review-Verfahren der Forschungsbeiträge wäre Forum Kinderund Jugendsport in dieser Form nicht möglich. Herzlichen Dank allen, die mit ihrer wichtigen Arbeit zum Gelingen dieser Zeitschrift beitragen:

- Tim Bindel (Mainz)

- Klaus Bös (Karlsruhe)

- Hans Peter Brandl-Bredenbeck (Augsburg)

- Dennis Dreiskämper (Münster)

- Jan Erhorn (Osnabrück)

- Norbert Gissel (Bochum)

- Marion Golenia (Münster)

- Rüdiger Heim (Heidelberg)

- David Jaitner (Braunschweig)

- Valerie Kastrup (Bielefeld)

- Miriam Kehne (Paderborn)
- Christa Kleindienst-Cachay (Bielefeld)

- Andreas Luh (Bochum)

- Stephan Maykus (Osnabrück)

- Katharina Morlang (Frankfurt am Main)

- Roland Naul (Münster)

- Miriam Seyda (Flensburg)

- Jessica Süßenbach (Lüneburg)

- Silvester Stahl (Potsdam)

- Andrea Szukala (Münster)

- Tobias Vogt (Köln)

- Torsten Weber (Köln)

- Bernd Wedemeyer-Kolwe (Hannover)

- Michaela Werkmann (Freiburg)

Wir wünschen Ihnen und Euch viele neue Informationen und Anregungen und viel Freude beim Lesen!

Stefan Raid (für die Institutionellen Herausgeber*innen)

Nils Neuber (für die Herausgeber*innen der Forschungsbeiträge)

Peter Lautenbach (für die Herausgeber*innen der Fachbeiträge)

\section{Korrespondenzadresse}

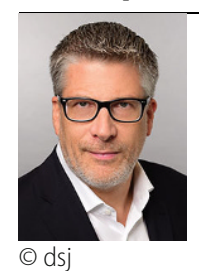

Stefan Raid

Deutsche Sportjugend Hamburg, Deutschland raid@dsj.de

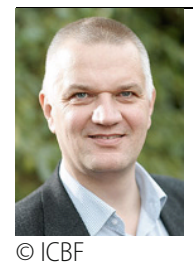

Prof. Dr. Nils Neuber

Westfälische WilhelmsUniversität Münster Münster, Deutschland nils.neuber@uni-muenster.de

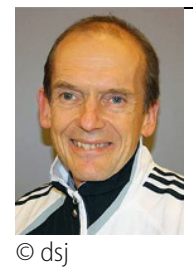

Peter Lautenbach

Deutsche Sportjugend

Frankfurt am Main,

Deutschland

lautenbach@dsj.de

Stefan Raid 1. Vorsitzender Deutsche Sportjugend

Prof. Dr. Nils Neuber Professor für Bildung und Unterricht im Sport
Peter Lautenbach ehemaliger Ressortleiter Deutsche Sportjugend

Funding. Open Access funding enabled and organized by Projekt DEAL.

Interessenkonflikt. S. Raid, N. Neuber und P. Lautenbach geben an, dass kein Interessenkonflikt besteht.

Open Access. Dieser Artikel wird unter der Creative Commons Namensnennung 4.0 International Lizenz veröffentlicht, welche die Nutzung, Vervielfältigung, Bearbeitung, Verbreitung und Wiedergabe in jeglichem Medium und Format erlaubt, sofern Sie den/die ursprünglichen Autor(en) und die Quelle ordnungsgemäß nennen, einen Link zur Creative Commons Lizenz beifügen und angeben, ob Änderungen vorgenommen wurden.

Die in diesem Artikel enthaltenen Bilder und sonstiges Drittmaterial unterliegen ebenfalls der genannten Creative Commons Lizenz, sofern sich aus der Abbildungslegende nichts anderes ergibt. Sofern das betreffende Material nicht unter der genannten Creative Commons Lizenz steht und die betreffende Handlung nicht nach gesetzlichen Vorschriften erlaubt ist, ist für die oben aufgeführten Weiterverwendungen des $\mathrm{Ma}$ terials die Einwilligung des jeweiligen Rechteinhabers einzuholen.

Weitere Details zur Lizenz entnehmen Sie bitte der Lizenzinformation auf http://creativecommons.org/ licenses/by/4.0/deed.de. 